\title{
A Conic Integer Programming Approach to Stochastic Joint Location-Inventory Problems
}

\author{
Alper Atamtürk, Gemma Berenguer, Zuo-Jun (Max) Shen \\ Department of Industrial Engineering and Operations Research, University of California, Berkeley, Berkeley, California 94720 \\ \{atamturk@ berkeley.edu,gemmabf@ berkeley.edu, shen@ieor.berkeley.edu\}
}

\begin{abstract}
We study several joint facility location and inventory management problems with stochastic retailer demand. In particular, we consider cases with uncapacitated facilities, capacitated facilities, correlated retailer demand, stochastic lead times, and multicommodities. We show how to formulate these problems as conic quadratic mixed-integer problems. Valid inequalities, including extended polymatroid and extended cover cuts, are added to strengthen the formulations and improve the computational results. Compared to the existing modeling and solution methods, the new conic integer programming approach not only provides a more general modeling framework but also leads to fast solution times in general.
\end{abstract}

Subject classifications: integrated supply chain; risk pooling; conic mixed-integer program; polymatroids; covers. Area of review: Transportation.

History: Received December 2010; revisions received July 2011, October 2011; accepted November 2011.

\section{Introduction}

To achieve significant cost savings across the supply chain, the major cost components that can impact the performance of the supply chain should be considered jointly rather than in isolation. This is not only true for decisions at the same hierarchical level (for instance, it is well known that the inventory management scheme and the transportation strategy should be integrated) but also at different levels.

Recently, we have seen a proliferation of research on integrated facility location and inventory management models. These models simultaneously consider decisions at both the strategic (location decisions) level and tactical (inventory decisions) level. Daskin et al. (2002) and Shen et al. (2003) were the first to propose joint location-inventory models with nonlinear safety stock costs and integer location decisions. The nonlinearity arises from the risk pooling strategy used to buffer random demand at the retailers. Specifically, they consider the design of a supply chain system in which a supplier ships products to a set of retailers, each with uncertain demand. The decision problem is to determine how many distribution centers to locate, where to locate them, which retailers to assign to each distribution center (DC), how often to reorder at the distribution center, and what level of safety stock to maintain to minimize total location, shipment, and inventory costs while ensuring a specified level of service.

The complexity of integrated models with integer decision variables and nonlinear costs and constraints suggested the development of special-purpose heuristic algorithms for various special cases. Shen et al. (2003) outline a column generation approach while Daskin et al. (2002) propose a Lagrangian relaxation approach for this problem. Both of the approaches utilize a low-order polynomial algorithm for solving a nonlinear (concave) integer subproblem. Özsen et al. (2008) study a capacitated version of the joint location-inventory problem, and they design an efficient algorithm to handle fractional terms in the objective function and nonlinear capacity constraints.

In this paper we propose a new flexible and general approach based on recent developments in conic integer programming. In particular, we reformulate the joint locationinventory models with different types of nonlinearities as conic quadratic mixed-integer programs, which can then be solved directly using standard optimization software packages without the need for designing specialized algorithms. This approach has several advantages over the Lagrangian relaxation and column generation approaches. For the later approaches to work well, one needs to design specialpurpose algorithms for solving the nonlinear sub-problems and, for their exact solutions, implement a specialized branch-and-bound algorithm that either makes use of the Lagrangian relaxation bounds or allows convenient generation of columns in the search tree. In many cases, this requires an extensive programming effort which often gives way to simpler heuristics approaches as alternative. Moreover, these special-purpose algorithms often work under simplifying assumptions on the problems and are not easily extendable to more general settings. On the other hand, as we will see in the later discussions, our proposed conic quadratic programming based approach is direct, efficient, and flexible enough to handle more general problems that have been considered before in the literature, including correlated retailer demand, stochastic lead times, and multicommodity cases. 
The main contributions in this paper can be summarized as follows:

1. We propose a new approach to modeling and solving integrated supply chain problems with stochastic demand. The conic integer programming based approach is general, flexible, and efficient.

2. We show how to reformulate different types of nonlinearities arising in joint location-inventory problems as conic quadratic integer programs.

3. We address for the first time the joint locationinventory problems with distinct variance-to-mean ratio for each retailer, correlated retailer demand, stochastic lead times, and correlated multicommodity demand.

4. We strengthen the conic quadratic integer formulations with cutting planes for their efficient solution.

5. We perform computational studies, comparing the new approach with earlier ones in the literature that deal with special cases of our general model, and investigate the impact of correlated demand on the supply chain design.

The rest of the paper is organized as follows. In \$2 we review the relevant literature on integrated location and inventory optimization and recent developments in conic programming. In $\$ 3$ we formally define a conic quadratic mixed-integer program and review the notation and parameters used in the paper. In $\$ 4$ we address the basic uncapacitated model and give a conic quadratic mixed-integer formulation for it. In $\$ 5$ we study the capacitated model and its respective equivalent conic mixed-integer reformulation. In these two sections, we also show how to utilize relevant polymatroid and cover inequalities for strengthening the conic quadratic formulations. In $\S 6$ we generalize the models to incorporate correlated retailer demand, stochastic lead times, and multicommodities. Each model is accompanied by its equivalent conic quadratic formulation. In $\$ 7$ we present our computational results with the conic quadratic MIP approach, provide comparisons with earlier studies, and investigate the impact of correlations and stochastic lead times. Finally, in $\$ 8$ we conclude with a few final remarks.

\section{Literature Review}

In this section we review the literature on integrated supply chain design models, especially the papers that model fixed location costs and nonlinear inventory costs. We mention some work related to multicommodity in supply chain design and retailers' and products' demand correlation. Recent developments on conic integer programming are also reviewed.

Daskin et al. (2002) and Shen et al. (2003) propose the first location-inventory model with nonlinear inventory costs. They propose Lagrangian relaxation and column generation methods for its solution, respectively. Both methods employ the same subproblem, which is solved in $O(n \log n)$ for two special cases: when the variance of the demand is proportional to the mean (as in the Poisson demand case), or when the demand is deterministic. In these cases the objective function simplifies to one with a single nonlinear (concave) term for each retailer, which underlies the efficient solution approach. Shu et al. (2005) and Shen and Qi (2007) study more general models in which these assumptions on demand are relaxed. As a result, multiple nonlinear terms appear in the objective functions. Specifically, Shu et al. (2005) study a subproblem with two concave terms and Shen and Qi (2007) added a third term to accommodate routing costs. More general problems are studied by Qi and Shen (2007), Shen (2005), Shen and Daskin (2005), and Snyder et al. (2007).

Özsen et al. (2008) consider the capacitated version of the models in Shen et al. (2003) and Daskin et al. (2002). They propose a Lagrangian relaxation based solution algorithm to solve the problem, where the Lagrangian subproblems are nonlinear integer program which include concave and fractional terms. For more detailed review on integrated location-inventory models, we refer the reader to Shen (2007).

Multicommodity problems have been studied in the location literature and are of our interest for the present paper. Geoffrion and Graves (1974) utilize a Bender's decomposition to solve multicommodity problems with capacitated plants and DCs. Dasci and Verter (2001) consider economies of scale by introducing concave technology selection cost into the objective function of a multicommodity location model. To handle concavity of the objective function, they solve the problem with a series of piecewise linear underestimations. In the integrated supply chain design literature, Shen (2005) presents a multicommodity model that includes economies of scale cost terms in the objective function. The author proposes a Lagrangian relaxation solution algorithm with a low-order polynomial algorithm to solve the Lagrangian relaxation subproblems.

Correlated demand has received much attention in the inventory management literature, and it can be studied across time, sites, and products. Johnson and Thompson (1975) are among the first to study correlated demand in a single item and a single location setting. Erkip et al. (1990) consider a multi-echelon inventory system where demand is a first-order autoregressive process and is correlated across sites and time. These authors solve for the optimal safety stock level and show the impact of demand correlation over time. Charnes et al. (1995) assume that the sequence of demand is a covariance-stationary Gaussian stochastic process. The literature on supply chain problems with correlation between different products is scarce. Inderfurth (1991) studies the effects of correlation between different items on the optimal safety stock in stochastic multi-stage production/distribution systems. Fine and Freund (1990) and Goyal and Netessine (2011) study the correlation between products in the context of product and volume flexibility.

Recently, there has been a number of advances in the theory of conic integer programming. Atamtürk and Narayanan (2010) give conic mixed-integer rounding inequalities for 
conic quadratic mixed-integer programs and Çezik and Iyengar (2005) give convex quadratic cuts for mixed 0-1 conic programs. Atamtürk and Narayanan (2011) propose lifting methods for conic mixed-integer programming. Atamtürk and Narayanan (2009) propose cover-type inequalities for submodular knapsack sets and Atamtürk and Narayanan (2008) introduce polymatroid inequalities that can help with solving special structured conic quadratic programs efficiently. We have utilized these recently introduced valid inequalities for the efficient solution of our joint location-inventory models.

\section{Preliminaries}

A conic quadratic mixed-integer program (CQMIP) is an optimization problem of the form

$\min c^{\prime} x$,

s.t. $\left\|A_{i} x+b_{i}\right\|_{2} \leqslant d_{i}^{\prime} x+e_{i}, \quad i=1, \ldots, p$,

where $x \in \mathbb{Z}^{n} \times \mathbb{R}^{m},\|\cdot\|_{2}$ is the Euclidean norm and all parameters are rational. Observe that a linear constraint can be written as a special case of a conic quadratic constraint by letting $A_{i}=b_{i}=0$. Similarly, a convex quadratic constraint can be written as a special case by letting $d_{i}^{\prime}=0$. For an introduction to (convex) conic quadratic programming we refer the reader to Ben-Tal and Nemirovski (2001) and Alizadeh and Goldfarb (2003). In recent years there have been significant developments on the computation of conic quadratic mixed-integer programs. Due to the rise in demand for solution of CQMIP, commercial optimization software vendors such as CPLEX and Mosek have added to their offerings branch-and-bound based solvers for CQMIP.

During the last decade, conic quadratic programs have been employed to solve problems in different areas such as portfolio optimization, scheduling, and energy planning. Indeed, basic uncapacitated facility location problems have been formulated as conic quadratic programs (e.g., Kuo and Mittelmann 2004). In this paper, we show how to model nonlinear mixed $0-1$ optimization models arising in complex supply chain design problems as conic quadratic mixed 0-1 programs and utilize the recent advances in cutting planes for their scalable solution.

The following parameters and notation are used throughout the paper:

\section{Demand}

$\mu_{i}$ : mean of daily demand at retailer $i$,

$\sigma_{i}$ : standard deviation of daily demand at retailer $i$,

$V$ : variance-covariance matrix of daily demand at retailers.

Costs

$d_{i j}$ : unit cost of shipment between retailers $i$ and $j$,

$f_{j}$ : annualized fixed cost of locating a DC at retailer site $j$,

$F_{j}$ : fixed cost of placing an order at DC $j$,

$a_{j}$ : unit cost of shipment from the central plant to $\mathrm{DC} j$, $g_{j}$ : fixed cost per shipment from the central plant to DC $j$, $h$ : unit inventory holding cost per year.

\section{Weights}

$\beta$ : weight associated with the transportation costs,

$\theta$ : weight associated with the inventory costs.

Other parameters

$\chi$ : days worked per year,

$\alpha$ : service level,

$z_{\alpha}$ : standard normal deviation associated with service level $\alpha$,

$L_{j}$ : lead time in days at $\mathrm{DC} j$.

Decision variables

$x_{j}= \begin{cases}1, & \text { if a distribution center (DC) is located at } \\ & \text { retailer site } j, \\ 0, & \text { otherwise }\end{cases}$

$y_{i j}=\left\{\begin{aligned} 1, & \text { if retailer } i \text { is assigned to DC located at } \\ & \text { retailer site } j, \\ 0, & \text { otherwise. }\end{aligned}\right.$

\section{Model with Uncapacitated Facilities}

We start with the basic uncapacitated location-inventory model, which was originally studied by Daskin et al. (2002) and Shen et al. (2003). Their model assumes the following:

- Shipments are direct from DCs to retailers.

- Demand at each retailer is independent and Gaussian.

- Each retailer is supplied from exactly one DC.

\subsection{Model 1}

Under the assumptions listed above, the joint locationinventory model is formulated as follows:

$$
\begin{gathered}
\min \sum_{j \in J}\left(f_{j} x_{j}+\sum_{i \in I} \hat{d}_{i j} y_{i j}+K_{j} \sqrt{\sum_{i \in I} \mu_{i} y_{i j}}\right. \\
\left.+q_{j} \sqrt{\sum_{i \in I} \sigma_{i}^{2} y_{i j}}\right)
\end{gathered}
$$

(P1)

$$
\begin{array}{ll}
\text { s.t. } & \sum_{j \in J} y_{i j}=1, \quad i \in I, \\
& y_{i j} \leqslant x_{j}, \quad i \in I, j \in J, \\
& x_{j}, y_{i j} \in\{0,1\}, \quad i \in I, j \in J,
\end{array}
$$

where $\hat{d}_{i j}=\beta \chi\left(d_{i j}+a_{j}\right) \mu_{i}, K_{j}=\sqrt{2 \theta h\left(F_{j}+\beta g_{j}\right) \chi}$, and $q_{j}=z_{\alpha} \theta \sqrt{L_{j}} h$.

The objective of (P1) is to minimize total expected cost of location, shipment, and inventory management. The first objective term is the fixed cost of locating DC $j, f_{j} x_{j}$. The second term is the cost of shipping from DC $j$ to the retailers and from the central plant to DC $j$, $\beta \chi \sum_{i \in I}\left(d_{i j}+a_{j}\right) \mu_{i} y_{i j}$. The third term captures the working inventory effects due to the fixed costs of placing orders 
and the fixed costs of shipping from the central plant to DC $j, \sqrt{2 \theta h\left(F_{j}+\beta g_{j}\right) \chi} \sqrt{\sum_{i \in I} \mu_{i} y_{i j}}$. The fourth term is the expected safety stock cost at DC $j, z_{\alpha} \theta \sqrt{L_{j}} h \sqrt{\sum_{i \in I} \sigma_{i}^{2} y_{i j}}$. Appendix A supplies the derivation of the working inventory term.

Constraints (1) ensure that each retailer is assigned to exactly one DC. Constraints (2) guarantee that retailers are only assigned to open DCs. Constraints (3) define the domain of the decision variables.

As mentioned in the literature review, in order to handle the nonlinearity of the objective, Shen et al. (2003) solve (P1) by transforming it into a set-covering model and solve it using column generation approach, where the columns are generated by solving an unconstrained nonlinear subproblem on binary variables. Daskin et al. (2002) solve the same problem by designing a Lagrangian relaxation algorithm. In both of these papers the ratio of the demand variance to the mean demand is assumed to be constant for all retailers $\left(\sigma_{i}^{2} / \mu_{i}=\gamma \forall i\right)$. Under this assumption, (P1) would have only one square root term instead of two for each retailer, which makes the Lagrangian and column generation subproblems easier to solve. Our approach does not require this assumption.

\subsection{A Conic Quadratic MIP Formulation}

In this section we show how to reformulate (P1) as a conic quadratic mixed-integer program (CQMIP). The advantage of the CQMIP formulation is that it can be solved directly using standard optimization software packages such as CPLEX or Mosek.

By introducing auxiliary variables $t_{1 j}, t_{2 j} \geqslant 0$ to represent the nonlinear terms in the objective and using the fact that $y_{i j}=y_{i j}^{2}$, we reformulate $(\mathrm{P} 1)$ as

$$
\begin{aligned}
& \min \sum_{j \in J}\left(f_{j} x_{j}+\sum_{i \in I} \hat{d}_{i j} y_{i j}+K_{j} t_{1 j}+q_{j} t_{2 j}\right), \\
& \text { (CQMIP1) s.t. } \sum_{i \in I} \mu_{i} y_{i j}^{2} \leqslant t_{1 j}^{2}, \quad j \in J \text {, } \\
& \sum_{i \in I} \sigma_{i}^{2} y_{i j}^{2} \leqslant t_{2 j}^{2}, \quad j \in J, \\
& t_{1 j}, t_{2 j} \geqslant 0, \quad j \in J,
\end{aligned}
$$$$
\text { (1)-(3). }
$$

Note that the objective of (CQMIP1) is linear and the constraints are either conic quadratic or linear, which fits into the general conic quadratic mixed-integer programming model described in $\S 3$.

\subsection{Polymatroid Inequalities}

Commercial software packages utilize a branch-and-bound algorithm for solving conic quadratic MIPs, and their performance can be significantly improved by strengthening the formulations with structural cutting planes. In this section, utilizing submodularity, we will reformulate constraints (4) and (5) with polymatroid inequalities of Atamtürk and Narayanan (2008) to strengthen the convex relaxation of CQMIP1.

Definition 1. A function $g: 2^{I} \rightarrow \mathbb{R}$ is submodular if $g(S \cup i)-g(S) \geqslant g(T \cup i)-g(T)$ for all $S \subseteq T \subseteq I$ and $i \in I \backslash T$.

Definition 2. For a submodular function $g$, the polyhedron

$E P_{g}=\left\{\pi \in \mathbb{R}^{I}: \pi(S) \leqslant g(S), \forall S \subseteq I\right\}$

is called an extended polymatroid.

For an extended polymatroid $E P_{g}$, Atamtürk and Narayanan (2008) show that the linear inequality

$\pi y \leqslant t \quad$ with $\quad \pi \in E P_{g}$

is valid for the lower convex envelope of $g$ :

$Q_{g}:=\operatorname{conv}\left\{(y, t) \in\{0,1\}^{|I|} \times \mathbb{R}: g(y) \leqslant t\right\}$.

Because $t_{j} \geqslant 0, \forall j \in J$ and $y_{i j}^{2}=y_{i j}, \forall i \in I, j \in J$, inequalities $\sum_{i \in I} \mu_{i} y_{i j}^{2} \leqslant t_{j}^{2}$ and $\sqrt{\sum_{i \in I} \mu_{i} y_{i j}} \leqslant t_{j}$ are equivalent. The latter inequalities have a submodular form due to the concavity of the square root function and the nonnegativity of the arguments in the square root function. More precisely, the set function

$g(S):=\sqrt{\sum_{i \in S} \mu_{i}}, \quad \forall S \subseteq I$

is submodular.

Although there are exponentially many extremal (corresponding to extreme points $\pi$ of $E P_{g}$ ) extended polymatroid inequalities, only a small subset of them is needed in the branch-and-bound search tree. It turns out that, given a solution, finding a violated polymatroid cut can be done easily. Formally, the separation problem for the extended polymatroid inequalities is defined as follows:

Given $\left(y^{*}, t^{*}\right) \in[0,1]^{|I|} \times \mathbb{R}_{+}$, let

$\zeta=\max \left\{\pi y^{*}: \pi \in E P_{g}\right\}$

If $\zeta>t^{*}$, then the extended polymatroid inequality $\pi^{*} x \leqslant t$ for an optimal $\pi^{*}$ cuts off $\left(y^{*}, t^{*}\right)$; otherwise, there exists no violated extended polymatroid inequality. Thus, the separation problem is an optimization over an extended polymatroid, which is solved by the greedy algorithm of Edmonds (1970). For completeness we describe the algorithm in Appendix B. 


\section{Model with Capacitated Facilities}

In this section we consider the generalization of (P1) with facility capacities and show how to reformulate it as a conic quadratic MIP. Özsen et al. (2008) present a generalization of the integrated inventory-location model (P1) by introducing inventory capacity constraints for the DCs. These constraints are defined for a $(Q, r)$ inventory control policy with type-I service level. Compared with model (P1), their model contains additional nonlinear terms:

- Nonlinear (concave) capacity constraints for each DC,

- Nonlinear (fractional) terms in the objective function.

As in the uncapacitated counterparts of Daskin et al. (2002) and Shen et al. (2003), in order to simplify the problem, Özsen et al. (2008) also assume the variance of each retailer's demand to be proportional to the mean demand. In particular, $\sigma_{i}^{2}=\mu_{i} \forall i$. We do not make this assumption here.

\subsection{Model 2}

Let $C_{j}$ be the maximum inventory capacity of DC $j$ and $Q_{j}$ be the reorder quantity for DC $j$. Then, the integrated inventory-location model with capacitated facilities is formulated as the following nonlinear mixed 0-1 optimization problem:

(P2)

$$
\begin{array}{ll}
\min \sum_{j \in J}\left(f_{j} x_{j}+\sum_{i \in I} \hat{d}_{i j} y_{i j}+\hat{F}_{j} \frac{\sum_{i \in I} \mu_{i} y_{i j}}{Q_{j}}\right. & \\
& \left.+q_{j} \sqrt{\sum_{i \in I} \sigma_{i}^{2} y_{i j}}+\theta h \frac{Q_{j}}{2}\right), \\
\text { s.t. } Q_{j} & +z_{\alpha} \sqrt{L_{j}} \sqrt{\sum_{i \in I} \sigma_{i}^{2} y_{i j}}+L_{j} \sum_{i \in I} \mu_{i} y_{i j} \leqslant C_{j}, \\
& Q_{j} \geqslant 0, \quad j \in J, \\
& (1)-(3),
\end{array}
$$

where $\hat{F}_{j}=\left(F_{j}+\beta g_{j}\right) \chi$.

The third term in the objective is the expected fixed cost of placing an order at DC $j$ and the expected fixed cost per shipment from the central plant to DC $j$. The fifth term is new; it is the average inventory holding cost at DC $j$.

Constraints (7) define the capacity of each DC to be the sum of the order quantity $Q_{j}$ and the reorder point. Note that in defining the DC capacity, we consider the worst-case scenario, i.e., no demand is observed during lead time. The reorder point is the sum of the safety stock, $z_{\alpha} \sqrt{L_{j}} \sqrt{\sum_{i \in I} \sigma_{i}^{2} y_{i j}}$, and the expected demand during lead time, $L_{j} \sum_{i \in I} \mu_{i} y_{i j}$.

\subsection{An Equivalent Conic Quadratic MIP Model}

The objective of (P2) is neither concave nor convex. Özsen et al. (2008) develop a Lagrangian relaxation based heuristic algorithm to solve this problem. In this section we show how to transform $(\mathrm{P} 2)$ into the following equivalent conic quadratic MIP, which leads to an exact solution of the problem. Consider

\section{(CQMIP2)}

$$
\begin{array}{ll}
\min & \sum_{j \in J}\left(f_{j} x_{j}+\sum_{i \in I} \hat{d}_{i j} y_{i j}+q_{j} t_{j}+\frac{\theta}{2} h z_{j}\right), \\
\text { s.t. } & Q_{j}+z_{\alpha} \sqrt{L_{j}} t_{j}+L_{j} \sum_{i \in I} \mu_{i} y_{i j} \leqslant C_{j} x_{j}, \quad j \in J, \\
& \sum_{i \in I} \sigma_{i}^{2} y_{i j}^{2} \leqslant t_{j}^{2}, \quad \forall j \in J, \\
& \sum_{i \in I} H_{j} \mu_{i} y_{i j}^{2}+\left(Q_{j}-\frac{z_{j}}{2}\right)^{2}-\frac{z_{j}^{2}}{4} \leqslant 0, \quad j \in J, \\
& t_{j}, z_{j} \geqslant 0, \quad j \in J,
\end{array}
$$

(1)-(3), (8),

where $H_{j}=\hat{F}_{j} /((\theta h) / 2)$.

Constraints (1)-(3), and (8) are still present in the transformed problem. Constraints (7) are linearized as (9). An auxiliary variable $t_{j}$ is introduced for each $j$ and defined by the constraints (10). Constraints (9) have stronger right hand sides than constraints (7). We linearize the objective by using $t_{j}$ and the auxiliary variables for $z_{j}$ for each $j$. Variables $z_{j}$ are defined by the constraints (11) and (12).

Proposition 1. Problem (P2) is equivalent to (CQMIP2).

Proof. Variables $t_{j}$ and constraint (10) are used to substitute the terms $\sqrt{\sum_{i \in I} \sigma_{i}^{2} y_{i j}}$ as in (MIPCQ1). The second substitution for the third and fifth inventory terms $\left(\sum_{i \in I} \mu_{i} y_{i j}\right) / Q_{j}+Q_{j}$ follows from the following identities:

$$
\begin{aligned}
\frac{\sum_{i \in I} \mu_{i} y_{i}}{Q}+Q \leqslant z & \Leftrightarrow \sum_{i \in I} \mu_{i} y_{i}+Q^{2} \leqslant Q z \quad(\text { as } Q>0) \\
& \Leftrightarrow \sum_{i \in I} \mu_{i} y_{i}^{2}+Q^{2} \leqslant Q z \quad\left(\text { as } y_{i}=y_{i}^{2}\right) \\
& \Leftrightarrow \sum_{i \in I} \mu_{i} y_{i}^{2}+\left(Q-\frac{z}{2}\right)^{2} \leqslant \frac{z^{2}}{4} .
\end{aligned}
$$

\subsection{Extended Cover Cuts}

To strengthen formulation (CQMIP2) we add cover type inequalities derived from nonlinear knapsack relaxations of the formulation. Toward this end, consider the capacity constraints (9). For each $j$, we relax the left-hand side of the constraint by dropping $Q_{j}$. Furthermore, we substitute $t_{j}$ with the left-hand side of constraint (10) to arrive at the nonlinear 0-1 knapsack constraint

$z_{\alpha} \sqrt{L_{j}} \sqrt{\sum_{i \in I} \sigma_{i}^{2} y_{i j}}+L_{j} \sum_{i \in I} \mu_{i} y_{i j} \leqslant C_{j}$.

For simplicity of notation, we drop the subscript $j$ to define the inequalities. For inequality (13), define the set function $f: 2^{I} \rightarrow \mathbb{R}$, where

$f(S)=z_{\alpha} \sqrt{L} \sqrt{\sigma^{2}(S)}+L \mu(S)$, 
$\sigma^{2}(S):=\sum_{i \in S} \sigma_{i}^{2}$ and $\mu(S):=\sum_{i \in S} \mu_{i}$. Using submodularity of $f$, Atamtürk and Narayanan (2009) give cover and extended cover cuts for the submodular knapsack set,

$$
\begin{aligned}
Y & =\left\{y \in\{0,1\}^{|I|}: f(y) \leqslant C\right\} \\
& =\left\{y \in\{0,1\}^{|I|}: z_{\alpha} \sqrt{L} \sqrt{\sum_{i \in I} \sigma_{i}^{2} y_{i}}+L \sum_{i \in I} \mu_{i} y_{i} \leqslant C\right\} .
\end{aligned}
$$

They show that given a subset of indices $S \subseteq I$ and the conic quadratic 0-1 knapsack set $Y$, we can find valid cover inequalities that depend on the cover set.

Definition 3. $S \subseteq I$ is called a cover for $Y$ if $z_{\alpha} \sqrt{L} \sqrt{\sigma^{2}(S)}+L \mu(S)>C$.

Atamtürk and Narayanan (2009) show that for cover $S$ the corresponding cover inequality

$\sum_{i \in S} y_{i} \leqslant|S|-1$

is valid for $Y$.

As with polymatroid inequalities, a separation algorithm generates the cover constraints at the root node of the branch-and-bound tree for each $j$. Given $y^{*} \in[0,1]^{|I|}$ a violated cover inequality can be found by solving the following nonlinear 0-1 separation problem:

$\zeta=\min \left\{\bar{y}^{\prime} z: z_{\alpha} \sqrt{L} \sqrt{\sum_{i \in I} \sigma_{i}^{2} z_{i}}+L \sum_{i \in I} \mu_{i} z_{i}>C, z \in\{0,1\}^{|I|}\right\}$,

where $\bar{y}=1-y^{*}$. If $\zeta<1$, then the cover inequality corresponding to optimal $z$ cuts off $y^{*}$. We employ a heuristic algorithm based on rounding the convex relaxation of the separation as proposed in Atamtürk and Narayanan (2009). For completeness, this algorithm is stated in Appendix C.

Cover inequalities can be strengthened by extending them with noncover variables. To introduce extended cover inequalities, we first need to define the difference function and the notion of extension.

Definition 4. Given a set function $f$ on $I$ and $i \in I$, the difference function $\rho$ is defined as $\rho_{i}(S):=f(S \cup i)-f(S)$ for $S \subseteq I \backslash i$.

Definition 5. Let $\pi=\left(k_{(1)}, \ldots, k_{(|I|-|S|)}\right)$ be a permutation of the indices in $I \backslash S$. Define $S_{\ell}=S \cup\left\{k_{(1)}, \ldots, k_{(\ell)}\right\}$ for $\ell=1, \ldots,|I|-|S|$, where $S_{0}=S$. The extension of $S$ corresponding to permutation $\pi$ is

$$
\begin{aligned}
& E_{\pi}(S):=S \cup U_{\pi}(S) \text {, where } \\
& \qquad U_{\pi}(S)=\left\{k_{(\ell)}: \rho_{k_{(\ell)}}\left(S_{\ell-1}\right) \geqslant \rho_{i}(\varnothing) \forall i \in S\right\} .
\end{aligned}
$$

Atamtürk and Narayanan (2009) also show that for given cover $S$ and permutation $\pi$, the corresponding extended cover inequality

$$
\sum_{i \in E_{\pi}(S)} y_{i} \leqslant|S|-1
$$

is valid for $Y$. We utilize extended cover inequalities in our computations presented in $\S 7$.

\section{Generalized Models}

In this section, we exploit the expressive power of conic programming to present more general integrated locationinventory models than considered to date. In addition to facility capacities, which have been introduced in the past, we now consider realistic aspects such as correlation between retailers' demand, stochastic lead times, and multicommodities.

\subsection{Model 3: Correlated Demands}

Let the retailer demand be a multinormal random variable with mean $\mu$ and variance-covariance matrix $V$. Generalizing the safety stock term in the previous section, in the presence of demand correlation, the safety stock at DC $j$ can be stated as $z_{\alpha} \sqrt{L_{j}} h \sqrt{y_{. j}^{\prime} V y_{. j}}$, where $y_{. j}$ is the assignment decision vector for the $j$ th DC.

The mathematical model for the correlated demand is the same as (P2) except that the variance terms are replaced with the more general variance-covariance matrix:

$$
\begin{gathered}
\min \sum_{j \in J}\left(f_{j} x_{j}+\sum_{i \in I} \hat{d}_{i j} y_{i j}+\hat{F}_{j} \frac{\sum_{i \in I} \mu_{i} y_{i j}}{Q_{j}}\right. \\
\left.+q_{j} \sqrt{y_{. j}^{\prime} V y_{. j}}+\theta h \frac{Q_{j}}{2}\right), \\
\text { s.t. } \quad Q_{j}+z_{\alpha} \sqrt{L_{j}} \sqrt{y_{. j}^{\prime} V y_{. j}}+L_{j} \sum_{i \in I} \mu_{i} y_{i j} \leqslant C_{j} x_{j}, \\
\qquad j \in J,
\end{gathered}
$$

(1)-(3), (8).

As in CQMIP2, we formulate (P3) by introducing auxiliary variables and linearizing the objective as a conic quadratic mixed 0-1 program:

(CQMIP3)

$$
\begin{aligned}
\min & \sum_{j \in J}\left(f_{j} x_{j}+\sum_{i \in I} \hat{d}_{i j} y_{i j}+q_{j} t_{j}+\frac{\theta}{2} h z_{j}\right), \\
\text { s.t. } & \sqrt{y_{. j}^{\prime} V y_{. j}} \leqslant t_{j}, \quad j \in J, \\
& (1)-(3),(8),(9),(11),(12) .
\end{aligned}
$$

\subsection{Model 4: Stochastic Lead Times}

In a real-life setting orders at DCs might arrive before or after the expected receiving time. Hence, in addition to correlated demand, a realistic aspect to be considered is stochastic lead times. To illustrate this situation, we define lead time between the central warehouse and each DC $j$ as a normal distribution with mean $L_{j}$ and standard deviation $\sigma_{L_{i}}$. We assume that successive lead times are independent and orders do not cross (Nahmias 1993).

Lead time variability and correlated demands affect the amount of safety stock at the DC level. In particular, we define the safety stock as follows:

$z_{\alpha} h \sqrt{L_{j} \sigma_{D_{j}}^{2}+\sigma_{L_{j}}^{2} \mu_{D_{j}}^{2}}$ 
where the variance of the demand at DC $j$ is $\sigma_{D_{j}}^{2}=y_{. j}^{\prime} V y_{. j}$ and the average demand at DC $j$ is $\mu_{D_{j}}=\sum_{i \in I} \mu_{i} y_{i j}$. Thus, $\mu_{D_{j}}^{2}=y_{. j}^{\prime} M y_{. j}$ where

$M=\left(\begin{array}{cccc}\mu_{1}^{2} & \mu_{1} \mu_{2} & \ldots & \mu_{1} \mu_{|I|} \\ \mu_{2} \mu_{1} & \mu_{2}^{2} & \ldots & \mu_{2} \mu_{|I|} \\ \vdots & \vdots & & \vdots \\ \mu_{|I|} \mu_{1} & \mu_{|I|} \mu_{2} & \cdots & \mu_{|I|}^{2}\end{array}\right)$.

With this notation, the integrated inventory-location model with capacitated facilities, correlated demand, and stochastic lead times is then formulated as the following problem:

$$
\begin{aligned}
\min \sum_{j \in J}( & f_{j} x_{j}+\sum_{i \in I} \hat{d}_{i j} y_{i j}+\hat{F}_{j} \frac{\sum_{i \in I} \mu_{i} y_{i j}}{Q_{j}} \\
& \left.+\tilde{q}_{j} \sqrt{y_{. j}^{\prime}\left(L_{j} V+\sigma_{L_{j}}^{2} M\right) y_{. j}}+\theta h \frac{Q_{j}}{2}\right),
\end{aligned}
$$

(P4)

$$
\begin{aligned}
\text { s.t. } \quad Q_{j} & +z_{\alpha} \sqrt{y_{. j}^{\prime}\left(L_{j} V+\sigma_{L_{j}}^{2} M\right) y_{. j}} \\
& +L_{j} \sum_{i \in I} \mu_{i} y_{i j} \leqslant C_{j} x_{j}, \quad j \in J
\end{aligned}
$$

$$
\text { (1)-(3), (8), }
$$

where $\tilde{q}_{j}=z_{\alpha} \theta h$.

The equivalent conic quadratic MIP (CQMIP4) is derived by employing the same substitution technique used for (CQMIP2):

$$
\min \sum_{j \in J}\left(f_{j} x_{j}+\sum_{i \in I} \hat{d}_{i j} y_{i j}+\tilde{q}_{j} t_{j}+\frac{\theta}{2} h z_{j}\right),
$$

(CQMIP4) s.t. $Q_{j}+z_{\alpha} t_{j}+L_{j} \sum_{i \in I} \mu_{i} y_{i j} \leqslant C_{j} x_{j}$,

$$
j \in J,
$$$$
\sqrt{y_{. j}^{\prime}\left(L_{j} V+\sigma_{L_{j}}^{2} M\right) y_{. j}} \leqslant t_{j}, \quad j \in J,
$$

$$
\text { (1)-(3), (8), (11), (12). }
$$

\subsection{Model 5: Multiple Commodities}

Because our models exhibit economies of scale terms, a multicommodity extension is of interest. Each commodity represents a specific product or product group, and we employ the subindex $l \in L$ to refer to different commodities. Before introducing the model, we need to define some new notation that depends on the type of commodity:

\section{Demand}

$\mu_{i l}$ : mean of daily demand at retailer $i$ for commodity $l$,

$\sigma_{i l}$ : standard deviation of daily demand at retailer $i$ for commodity $l$.

Costs

$d_{i j l}$ : cost per unit to ship commodity $l$ between retailers $i$ and $j$,
$F_{j l}$ : fixed cost of placing an order at DC $j$ for commodity $l$,

$a_{j l}:$ unit cost of shipment from the central plant to DC $j$ for commodity $l$,

$g_{j l}:$ fixed cost per shipment from the central plant to $\mathrm{DC} j$ for commodity $l$,

$h_{l}$ : unit inventory holding cost per unit of commodity $l$ per year.

Other parameters

$z_{\alpha^{\prime}}$ : standard normal deviation associated with service level of commodity $l, \alpha^{l}$,

$L_{j l}$ : lead time in days at DC $j$ for commodity $l$,

$\sigma_{L_{j l}}$ : standard deviation of lead time in days at DC $j$ for commodity $l$.

Decision variables

$y_{i j l}=\left\{\begin{aligned} 1, & \begin{array}{r}\text { if demand for commodity } l \text { of retailer } i \text { is } \\ \text { assigned to DC at retailer site } j,\end{array} \\ 0, & \text { otherwise. }\end{aligned}\right.$

$Q_{j l}:$ reorder quantity for DC $j$ of commodity $l$.

Under the notation defined above, the multicommodity joint location-inventory model with capacitated facilities, stochastic lead times, and correlated retailers' demand is formulated as follows:

$$
\begin{aligned}
& \min \sum_{j \in J}\left(f_{j} x_{j}+\sum_{l \in L}\left(\sum_{i \in I} \hat{d}_{i j l} y_{i j l}+\hat{F}_{j l} \frac{\sum_{i \in I} \mu_{i l} y_{i j l}}{Q_{j l}}\right.\right. \\
& \left.\left.\quad+\tilde{q}_{j l} \sqrt{y_{. j l}^{\prime}\left(L_{j l} V_{l}+\sigma_{L_{j l}}^{2} M_{l}\right) y_{. j l}}+\theta h_{l} \frac{Q_{j l}}{2}\right)\right), \\
& \text { s.t. } \sum_{l \in L}\left(Q_{j l}+z_{\alpha^{l}} h_{l} \sqrt{y_{. j l}^{\prime}\left(L_{j l} V_{l}+\sigma_{L_{j l}}^{2} M_{l}\right) y_{. j l}}\right. \\
& \left.\quad+L_{j l} \sum_{i \in I} \mu_{i l} y_{i j l}\right) \leqslant C_{j} x_{j}, \quad j \in J \\
& \sum_{j \in J} y_{i j l}=1, \quad i \in I, l \in L, \\
& y_{i j l} \leqslant x_{j}, \quad i \in I, j \in J, l \in L, \\
& x_{j}, y_{i j l} \in\{0,1\}, Q_{j l} \geqslant 0, \quad i \in I, j \in J, l \in L
\end{aligned}
$$

where $\hat{d}_{i j l}=\beta \chi\left(d_{i j l}+a_{j l}\right), \quad \hat{F}_{j l}=\left(F_{j l}+\beta g_{j l}\right) \chi, \quad \tilde{q}_{j l}=$ $z_{\alpha^{l}} \theta h_{l}$,

$y_{. j l}=\left(\begin{array}{c}y_{1 j l} \\ \vdots \\ y_{I j l}\end{array}\right)$,

$V_{l}$ is the variance-covariance matrix of retailers' demand related to commodity $l$, and

$M_{l}=\left(\begin{array}{cccc}\mu_{1 l}^{2} & \mu_{1 l} \mu_{2 l} & \ldots & \mu_{1 l} \mu_{|I| l} \\ \mu_{2 l} \mu_{1 l} & \mu_{2 l}^{2} & \ldots & \mu_{2 l} \mu_{|I| l} \\ \vdots & \vdots & & \vdots \\ \mu_{|I| l} \mu_{1 l} & \mu_{|I| l} \mu_{2 l} & \ldots & \mu_{|I| l}^{2}\end{array}\right)$. 
Consequently, we have the following the conic quadratic reformulation of (P5):

\section{(CQMIP5)}

$$
\begin{array}{ll}
\min & \sum_{j \in J}\left(f_{j} x_{j}+\sum_{l \in L}\left(\sum_{i \in I} \hat{d}_{i j l} y_{i j l}+\tilde{q}_{j l} t_{j l}+\frac{\theta}{2} h_{l} z_{j l}\right)\right), \\
\text { s.t. } & \sum_{l \in L}\left(Q_{j l}+t_{j l}+L_{j l} \sum_{i \in I} \mu_{i l} y_{i j l}\right) \leqslant C_{j} x_{j}, \quad j \in J, \\
& \sqrt{y_{. j l}^{\prime}\left(L_{j l} V_{l}+\sigma_{L_{j l}}^{2} M_{l}\right) y_{. j l}} \leqslant t_{j l}, \quad j \in J, l \in L, \\
& \sum_{i \in I} H_{j l} \mu_{i} y_{i j l}^{2}+\left(Q_{j l}-\frac{z_{j l}}{2}\right)^{2}-\frac{z_{j l}^{2}}{4} \leqslant 0, \\
& t_{j l}, z_{j l} \geqslant 0, \quad j \in J, l \in L, \\
& (20)-(22),
\end{array}
$$

where $H_{j l}=\hat{F}_{j l} /\left(\left(\theta h_{l}\right) / 2\right)$.

\subsection{Model 6: Multiple Commodities with Correlated Demand}

In this last model, we consider the correlation among the demand of different commodities. Under this situation and for the simplicity of notation, we assume that the correlation coefficients related to commodities' demand are retailer-independent, and they are defined as $\rho_{l_{1} l_{2}}$ $\forall l_{1}, l_{2} \in L$. Similarly, the correlation coefficients of retailers' demand are commodity-independent and defined as $\rho_{i_{1} i_{2}} \forall i_{1}, i_{2} \in I$. Furthermore, we assume that the service level, inventory cost, and lead time parameters are the same regardless of commodity type (i.e., $z_{\alpha^{l}}=z_{\alpha}, h_{l}=h, L_{j l}=$ $L_{j}$, and $\left.\sigma_{L_{i l}}^{2}=\sigma_{L_{j}}^{2} \forall l \in L\right)$.

Under the notation defined above, the multicommodity joint location-inventory model with capacitated facilities, stochastic lead times, and correlated retailer and commodity demand is formulated as follows:

$$
\begin{aligned}
& \min \sum_{j \in J}( f_{j} x_{j}+\sum_{l \in L}\left(\sum_{i \in I} \hat{d}_{i j l} y_{i j l}+\hat{F}_{j l} \frac{\sum_{i \in I} \mu_{i l} y_{i j l}}{Q_{j l}}\right. \\
&\left.\left.+\theta h \frac{Q_{j l}}{2}\right)+\tilde{q}_{j} \sqrt{y_{. j .}^{\prime}\left(L_{j} U+\sigma_{L_{j}}^{2} W\right) y_{. j .}}\right),
\end{aligned}
$$

(P6) s.t. $\sum_{l \in L}\left(Q_{j l}+L_{j l} \sum_{i \in I} \mu_{i l} y_{i j l}\right)+z_{\alpha}$

$$
\cdot \sqrt{y_{. j .}^{\prime}\left(L_{j} U+\sigma_{L_{j}}^{2} W\right) y_{. j .}} \leqslant C_{j} x_{j}, \quad j \in J,
$$

$$
\text { (20)-(22), }
$$

where

$$
y_{. j .}=\left(\begin{array}{c}
y_{. j 1} \\
- \\
\vdots \\
- \\
y_{. j|L|}
\end{array}\right)
$$

is a block vector for the $j$ th DC,

$U=\left(\begin{array}{ccc}U_{11} & \ldots & U_{1|L|} \\ \vdots & & \vdots \\ U_{|L| 1} & \ldots & U_{|L| L \mid}\end{array}\right)$

is an $|L| \times|L|$ block matrix with $|I| \times|I|$ matrices

$U_{l_{1} l_{2}}=\rho_{l_{1} l_{2}}\left(\begin{array}{cccc}\sigma_{1 l_{1}} \sigma_{1 l_{2}} & \rho_{12} \sigma_{1 l_{1}} \sigma_{2 l_{2}} \ldots & \rho_{1|I|} \sigma_{1 l_{1}} \sigma_{|I| l_{2}} \\ \rho_{21} \sigma_{2 l_{1}} \sigma_{1 l_{2}} & \sigma_{2 l_{1}} \sigma_{2 l_{2}} & \ldots & \ldots \\ \vdots & \vdots & & \vdots \\ \rho_{|I| 1} \sigma_{|I| l_{1}} \sigma_{1 l_{2}} & \ldots & \ldots & \sigma_{|I| l_{1}} \sigma_{|I| l_{2}}\end{array}\right)$,

and

$W=\left(\begin{array}{ccc}W_{11} & \ldots & W_{1|L|} \\ \vdots & & \vdots \\ W_{|L| 1} & \ldots & W_{|L||L|}\end{array}\right)$

is an $|L| \times|L|$ block matrix with $|I| \times|I|$ matrices

$W_{l_{1} l_{2}}=\left(\begin{array}{cccc}\mu_{1 l_{1}} \mu_{1 l_{2}} & \mu_{1 l_{1}} \mu_{2 l_{2}} & \ldots & \mu_{1 l_{1}} \mu_{I l_{2}} \\ \mu_{2 l_{1}} \mu_{1 l_{2}} & \mu_{2 l_{1}} \mu_{2 l_{2}} & \ldots & \ldots \\ \vdots & \vdots & & \vdots \\ \mu_{I l_{1}} \mu_{1 l_{2}} & \ldots & \ldots & \mu_{I l_{1}} \mu_{I l_{2}}\end{array}\right)$.

Model 6 is the most general model we consider in this paper, and due to the flexibility of conic quadratic MIP approach, we arrive at the following formulation using the same transformations employed in special cases presented earlier:

\section{(CQMIP6)}

$$
\begin{array}{ll}
\min & \sum_{j \in J}\left(f_{j} x_{j}+\sum_{l \in L}\left(\sum_{i \in I} \hat{d}_{i j l} y_{i j l}+\frac{\theta}{2} h z_{j l}\right)+\tilde{q}_{j} t_{j}\right), \\
\text { s.t. } & \sum_{l \in L}\left(Q_{j l}+L_{j} \sum_{i \in I} \mu_{i l} y_{i j l}\right)+t_{j} \leqslant C_{j} x_{j}, \quad j \in J, \\
& \sqrt{y_{. j .}^{\prime}\left(L_{j} U+\sigma_{L_{j}}^{2} W\right) y_{. j .}} \leqslant t_{j}, \quad j \in J, \\
& \sum_{i \in I} \tilde{H}_{j l} \mu_{i} y_{i j l}^{2}+\left(Q_{j l}-\frac{z_{j l}}{2}\right)^{2}-\frac{z_{j l}^{2}}{4} \leqslant 0, \\
& t_{j}, z_{j l} \geqslant 0, \quad j \in J, l \in L, \quad l \in L, \\
& (20)-(22),
\end{array}
$$

where $\tilde{H}_{j l}=\hat{F}_{j l} /((\theta h) / 2)$.

\subsection{Polymatroid Cuts}

The cuts proposed in $\S \S 4$ and 5 are also pertinent to the generalized models presented in this section. It is reasonable to assume that retailers' demand are positively correlated as they are typically affected in the same direction 
by economic factors. Then we may employ polymatroid inequalities by reformulating the models using new binary variables for the products of binary variables. In particular, for model 3 we can replace the products $y_{i_{1} j} y_{i_{2} j}$ with $w_{i_{1} i_{2} j}$ by introducing constraints

$$
\begin{aligned}
w_{i_{1} i_{2} l} \leqslant y_{i_{1} j}, w_{i_{1} i_{2} l} \leqslant y_{i_{2} j}, y_{i_{1} j}+y_{i_{2} j} \leqslant 1+w_{i_{1} i_{2} j}, & \\
& i_{1}, i_{2} \in I, j \in J .
\end{aligned}
$$

These constraints ensure that $w_{i_{1} i_{2} j}=1$ if and only if $y_{i_{1} j}=$ $y_{i_{2} j}=1$. Noting that $w_{i_{1} i_{2} j}$ is equivalent to $w_{i_{1} i_{2} j}^{2}$, the safety stock at location $j$ can now be written as

$z_{\alpha} \sqrt{L_{j}} h \sqrt{\sum_{\left(i_{1} i_{2}\right) \in I \times I} V_{i_{1} i_{2}} w_{i_{1} i_{2} j}^{2}}$.

Thus we replace (15) with (32) and

$\sqrt{\sum_{\left(i_{1} i_{2}\right) \in I \times I} V_{i_{1} i_{2}} w_{i_{1} i_{2} j}^{2}} \leqslant t_{j}, \quad j \in J$.

As constraints (33) define the following extended polymatroid

$E P_{g}=\left\{\pi \in \mathbb{R}^{|I| \times|I|}: \sum_{\left(i_{1} i_{2}\right) \in S} \pi_{i_{1} i_{2}} \leqslant \sqrt{\sum_{\left(i_{1} i_{2}\right) \in S} V_{i_{1} i_{2}}}, \forall S \subseteq I \times I\right\}$,

we can now generate polymatroid cuts from $E P_{g}$ in the same manner as in $\S 4.3$. Benefits of these cuts for model 3 are illustrated in $\S 7$.

\section{Computational Results}

In this section we present our computational results on solving the corresponding conic quadratic MIP formulations of the joint location-inventory problems discussed in the previous sections. We compare our results with the earlier approaches based on Lagrangian relaxation and column generation methods for the special cases. We also study the impact of facility capacities, stochastic lead times, and demand correlations on the solutions.

The numerical experiments in this paper use data from the 1990 U.S. Census described in Daskin (1995). We employ four different data sets: a 15-node, 25-node, 88-node, and 150 -node data set. The 15 -node data set reports the node demand (population) of the 15 most populous U.S. states. The 25-node data set reports the node demand of the 25 largest cities in the United States. The 88-node data set reports the demand of each of the lower 48 U.S. state capitals plus Washington DC and the 50 largest U.S. cities (eliminating duplicates). The 150-node data set reports the demand of the 150 most populous U.S. cities. All data sets can be downloaded from the site http://sitemaker.umich.edu/

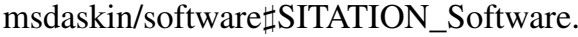

We use these data files in all our experiments except for those showing the computational benefits of adding cuts (Tables 4 and 5) and for those showing scalability for our most general model (Table 7), in which we report the averages for 10 randomly generated instances per row. Each random instance is generated by adding noise to the demand multiplying mean and standard deviation defined in the data files by $\left(1+\epsilon_{i}\right) \forall i$, where $\epsilon_{i}$ is drawn from Uniform $[-0.1,0.1]$. We also draw fixed cost from Uniform $[40,000,50,000]$. See Table D.1 in Appendix D for a summary of the parameter values used in all experiments. All computations are done on a 2.393-GHz Linux x86 computer using CPLEX 11.0.

\subsection{Numerical Experiments on the Uncapacitated Case}

To study the impact of inventory and transportation costs on the first model, we vary the values of $\beta$ and $\theta$, which are the weights of the transportation and inventory costs, respectively. We report computational results for different choices of $(\beta, \theta)$. Observing that when $\theta$ is larger than $\beta$, solution method require more time to arrive at optimality, we focus our attention on these cases. Higher values of $\theta$ assign more weight on the nonlinear terms of the objective terms.

For the experiments reported in Table 1, we use the 88- and 150-node data sets. For each run (row), we report the number of nodes (retailers), the transportation and inventory weights, the number of columns generated by the algorithm of Shen et al. (2003), and the corresponding CPU time, as well as the number of polymatroid cuts and CPU time of the conic integer programming approach.

So that we can directly compare the results, we ran the column generation method of Shen et al. (2003) and the conic integer model (CQMIP1) on the same computer using the same version of CPLEX. No branching was necessary for either approach for this data set. Indeed, as the polymatriod cuts define the convex hull of the nonlinear subproblem of Shen et al. (2003), both approaches give to the same relaxation values. We observe in this table that the conic method clearly outperforms the column generation method for both the 88 -node and 150 -node data sets. The aggregate times showed in Table 2 allow us to state that the proposed conic integer programming approach is fast and robust.

This experiment also provides managerial insight. When the inventory cost is relatively larger than the transportation cost, fewer DCs are opened in an optimal solution (observe DCs column in Table 1). Thus, under our model, a risk pooling strategy is favored when inventory costs are proportionally larger.

\subsection{Numerical Experiments on the Capacitated Case}

In Table 3, we report the results obtained by running conic integer program (CQMIP2) along with the results presented 
Table 1. Comparison with Shen et al. (2003).

\begin{tabular}{|c|c|c|c|c|c|c|c|}
\hline \multirow[b]{2}{*}{ Retailers } & \multirow[b]{2}{*}{$\beta$} & \multirow[b]{2}{*}{$\theta$} & \multirow[b]{2}{*}{ DCs } & \multicolumn{2}{|c|}{ Shen et al. (2003) set covering } & \multicolumn{2}{|c|}{ Conic formulation } \\
\hline & & & & Columns & Time & Cuts & Time \\
\hline 88 & 0.001 & 0.1 & 9 & 33,517 & 107 & 2 & 1 \\
\hline 88 & 0.002 & 0.1 & 11 & 19,686 & 24 & 5 & 1 \\
\hline 88 & 0.003 & 0.1 & 15 & 12,183 & 7 & 8 & 2 \\
\hline 88 & 0.004 & 0.1 & 21 & 8,907 & 3 & 6 & 2 \\
\hline 88 & 0.005 & 0.1 & 23 & 6,917 & 1 & 6 & 2 \\
\hline 88 & 0.001 & 0.1 & 9 & 33,517 & 107 & 3 & 1 \\
\hline 88 & 0.002 & 0.2 & 10 & 20,783 & 38 & 6 & 1 \\
\hline 88 & 0.005 & 0.5 & 22 & 7,868 & 2 & 18 & 3 \\
\hline 88 & 0.005 & 1 & 21 & 8,847 & 3 & 11 & 2 \\
\hline 88 & 0.005 & 5 & 17 & 12,628 & 7 & 30 & 5 \\
\hline 88 & 0.005 & 10 & 12 & 16,956 & 14 & 57 & 10 \\
\hline 88 & 0.005 & 20 & 9 & 27,899 & 51 & 161 & 30 \\
\hline 150 & 0.0004 & 0.01 & 15 & 45,551 & 226 & 20 & 12 \\
\hline 150 & 0.0006 & 0.01 & 21 & 23,767 & 89 & 15 & 8 \\
\hline 150 & 0.0008 & 0.01 & 26 & 14,239 & 49 & 11 & 11 \\
\hline 150 & 0.001 & 0.01 & 28 & 10,128 & 26 & 10 & 10 \\
\hline 150 & 0.0005 & 0.01 & 18 & 30,858 & 132 & 6 & 10 \\
\hline 150 & 0.001 & 0.02 & 28 & 10,778 & 26 & 13 & 11 \\
\hline 150 & 0.002 & 0.04 & 41 & 4,188 & 7 & 11 & 23 \\
\hline 150 & 0.001 & 0.01 & 28 & 10,128 & 26 & 10 & 10 \\
\hline 150 & 0.001 & 0.1 & 26 & 13,765 & 40 & 30 & 24 \\
\hline 150 & 0.001 & 0.5 & 21 & 23,397 & 78 & 66 & 56 \\
\hline 150 & 0.001 & 1 & 15 & 34,714 & 260 & 165 & 185 \\
\hline
\end{tabular}

in Özsen et al. (2008). We caution the reader that this table is descriptive, and we do not aim to directly compare the running time of the two approaches. The computations in Özsen et al. (2008) were done on a $1.7-\mathrm{GHz}$ computer, while we used a $2.393-\mathrm{GHz}$ computer. In addition, we employ CPLEX software, whereas computations in Özsen et al. (2008) are based on their own code written in $\mathrm{C}++$.

We report the run number, the number of retailers, the objective value, the number of nodes explored, and the CPU time for both approaches. Focusing on the results obtained by the conic integer programming approach, we observe that all our runs reach optimality fast. Some instances, such as with 150 retailers, do not even require any branching. Hence, we can state that our approach performs well in this experiment.

We have noticed that the facility capacities in the data set from Özsen et al. (2008) were often loose. To see the sensitivity of our approach to the tightness of facility capacities, we performed an additional experiment. Toward this end, we first created a problem instance where the capacity for each potential DC, $C$, was set to $19 \%$ of the total daily average demand, i.e., $\left(C / \sum_{i \in I} \mu_{i}\right) \times 100=19 \%$. Then we

Table 2. Summary statistic.

\begin{tabular}{lcc}
\hline Data set & $\begin{array}{c}\text { Aggregate time, } \\
\text { Shen et al. (2003) set covering }\end{array}$ & $\begin{array}{c}\text { Aggregate time, } \\
\text { Conic formulation }\end{array}$ \\
\hline 88-node & 364 & 60 \\
150-node & 959 & 360 \\
\hline
\end{tabular}

created additional problem instances by progressively tightening the DC capacity until reaching $16.287 \%$ (below this percentage the problem becomes infeasible). In Table 4 we report the CPU time in seconds and the number of nodes explored with and without adding cover and extended cover inequalities. We observe that problems generally become more difficult to solve as the capacity becomes tighter and that adding cover and extended cover inequalities reduces the solution times and the number of nodes significantly.

\subsection{Numerical Experiments on Correlated Retailer Demand Case}

Here we investigate the impact of correlated retailer demand on the joint location-inventory problem. First, to get an insight, we illustrate the effect of retailer demand correlations on a small example from the 25 -node set data using the parameter values listed in Table D.1. The dark links on Figure 1 show the retailer assignments in the optimal solution when there are no correlations. In this case four DCs are opened in New York, Los Angeles, Chicago, and Houston, and the expected total cost is 100,910. To see how correlations change the solution, we add correlation to the demand of the retailers served by Chicago and New York. Namely, we set the correlation between retailers Chicago, Detroit, Milwaukee, Indianapolis, and Columbus to $80 \%$ and similarly set the correlation between retailers New York, Philadelphia, Baltimore, and Washington to $80 \%$. This naturally increases the safety stock levels that need to be kept in Chicago and New York and the cost 
Table 3. Comparison with Özsen et al. (2008).

\begin{tabular}{|c|c|c|c|c|c|c|c|}
\hline \multirow[b]{2}{*}{ Problem } & \multirow[b]{2}{*}{ Retailers } & \multicolumn{3}{|c|}{ Özsen et al. (2008) Lagrangian } & \multicolumn{3}{|c|}{ Conic formulation } \\
\hline & & Objective & Nodes & Time & Objective & Nodes & Time \\
\hline 1 & 15 & 567,564 & 0 & 1 & 567,564 & 0 & 0 \\
\hline 2 & 15 & 595,707 & 1,154 & 11 & 595,707 & 0 & 0 \\
\hline 3 & 15 & 621,764 & 2,251 & 20 & 621,764 & 7 & 0 \\
\hline 4 & 15 & 630,051 & 4,207 & 37 & 630,051 & 9 & 0 \\
\hline 5 & 15 & 630,976 & 3,589 & 33 & 630,976 & 8 & 0 \\
\hline 6 & 15 & 642,722 & 68,943 & 618 & 642,722 & 37 & 0 \\
\hline 7 & 15 & $657,981^{*}$ & 83,033 & 743 & 653,361 & 98 & 0 \\
\hline 8 & 15 & 661,070 & 23,419 & 197 & 661,070 & 201 & 0 \\
\hline 9 & 15 & $668,430^{*}$ & 96,989 & 813 & 663,810 & 194 & 0 \\
\hline 10 & 15 & $987,298^{*}$ & 104,553 & 896 & 982,709 & 193 & 0 \\
\hline 11 & 88 & 322,627 & 0 & 3 & 322,627 & 0 & 1 \\
\hline 12 & 88 & 327,230 & 8,361 & 71 & 327,230 & 0 & 1 \\
\hline 13 & 88 & $328,702^{*}$ & 129,302 & 1,099 & 328,656 & 24 & 5 \\
\hline 14 & 88 & 328,808 & 37,400 & 329 & 328,808 & 3 & 5 \\
\hline 15 & 88 & 329,024 & 490,004 & 3,962 & 329,024 & 0 & 1 \\
\hline 16 & 88 & 330,900 & 79,566 & 696 & 330,900 & 6 & 4 \\
\hline 17 & 88 & 333,440 & 61,613 & 553 & 333,440 & 0 & 1 \\
\hline 18 & 88 & 337,911 & 87,412 & 765 & 337,911 & 2 & 2 \\
\hline 19 & 88 & $342,219^{*}$ & $1,612,263$ & 12,714 & 341,729 & 6 & 4 \\
\hline 20 & 88 & $344,845^{*}$ & 808,812 & 6,649 & 344,844 & 312 & 15 \\
\hline 21 & 150 & 468,645 & 0 & 10 & 468,645 & 0 & 3 \\
\hline 22 & 150 & 469,599 & 72,513 & 656 & 469,599 & 0 & 3 \\
\hline 23 & 150 & 469,740 & 95,129 & 824 & 469,740 & 0 & 3 \\
\hline 24 & 150 & 471,320 & 105,727 & 862 & 471,320 & 0 & 3 \\
\hline 25 & 150 & 473,743 & 177,718 & 1,457 & 473,743 & 0 & 4 \\
\hline 26 & 150 & 474,475 & 233,836 & 1,912 & 474,475 & 0 & 4 \\
\hline 27 & 150 & 474,750 & 307,282 & 2,485 & 474,750 & 0 & 4 \\
\hline 28 & 150 & 476,508 & 849,127 & 6,896 & 476,508 & 0 & 4 \\
\hline 29 & 150 & 477,314 & 462,340 & 3,783 & 477,314 & 0 & 4 \\
\hline 30 & 150 & 478,615 & 312,736 & 2,536 & 478,615 & 0 & 4 \\
\hline
\end{tabular}

*Not optimal.

of doing so. We see that in this case, the current solution is no longer optimal. Indeed, it is infeasible because the maximum capacity at the New York DC is smaller than the required inventory levels at this DC when accounting for correlated demands. The optimal solution (in light color) replaces the DC in Chicago with Indianapolis, and uncorrelated retailers that were served from New York and Houston are assigned to Indianapolis. Thus, as expected, the optimal solution pools more of the uncorrelated demand into the same DC and reduces pooling of correlated demand to keep the inventory levels and subsequent costs low. The expected total cost is 108,948 .

Table 4. Impact of capacities on solving (CQMIP2).

\begin{tabular}{|c|c|c|c|c|c|}
\hline \multirow{3}{*}{$\begin{array}{l}\text { DC capacity } \\
\text { (\% demand) }\end{array}$} & \multirow{2}{*}{\multicolumn{2}{|c|}{ CPLEX }} & \multicolumn{3}{|c|}{ CPLEX + Cuts } \\
\hline & & & \multirow[b]{2}{*}{ Time } & \multirow[b]{2}{*}{ Nodes } & \multirow{2}{*}{$\begin{array}{c}\text { Cover cuts } \\
\text { (ext cover cuts) }\end{array}$} \\
\hline & Time & Nodes & & & \\
\hline 19 & 67 & 4,080 & 85 & 3,060 & $1,562(0)$ \\
\hline 18.5 & 324 & 23,618 & 131 & 6,677 & $626(0)$ \\
\hline 18 & 202 & 10,219 & 169 & 9,999 & $588 \quad(0)$ \\
\hline 17.5 & 199 & 11,941 & 129 & 7,250 & $549(0)$ \\
\hline 17 & 400 & 27,794 & 167 & 9,402 & 483 (0) \\
\hline 16.5 & 64 & 2,848 & 64 & 2,480 & 576 (103) \\
\hline 16.35 & 140 & 7,621 & 119 & 5,340 & 574 (104) \\
\hline 16.3 & 90 & 5,419 & 82 & 3,668 & 575 (104) \\
\hline 16.29 & 133 & 6,603 & 102 & 5,523 & 576 (104) \\
\hline 16.287 & $2,033^{*}$ & $161,154^{*}$ & 764 & 31,936 & 577 (106) \\
\hline
\end{tabular}

*Instance could not be solved in 2,000 seconds. 
Figure 1. The effect of correlated retailer demand on the supply chain.

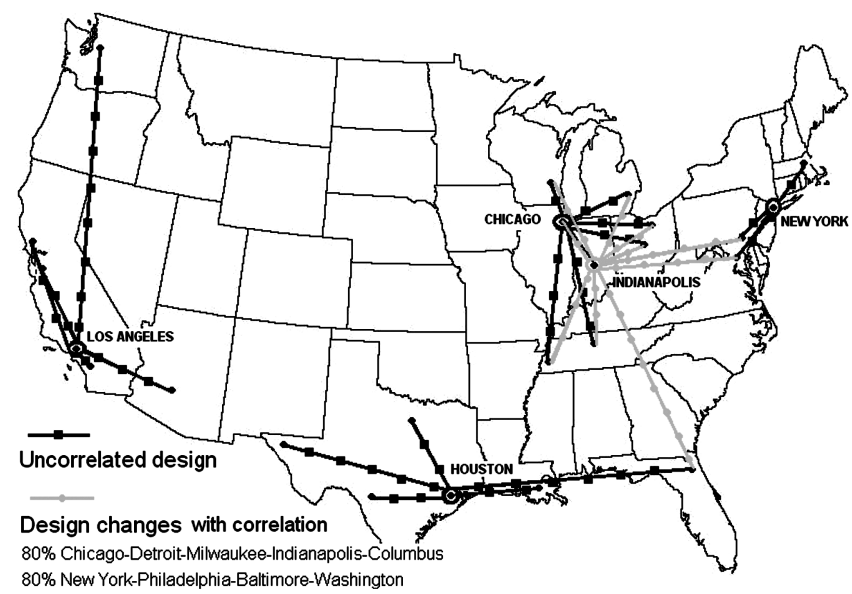

Continuing with this case, to see how correlations affect the total expected cost, this time we introduce positive correlation between every pair of retailers. To investigate the impact of correlations independent from capacity considerations and high cost of facility installations, we also set the DC capacities to a very large number and reduce the annualized facility fixed cost from 100,000 to 1,000 . Figure 2 shows the total expected cost as well as the number of DCs opened as a function of the retailer demand correlation. The expected total cost increases monotonically from 46,095 to 50,297 as more safety stock is needed in response to increasing demand correlation. Moreover, additional DCs are opened to reduce the number of retailers supplied by the same DC.

Finally, in Table 5 we report on the computational efficiency of solving (CQMIP3) with and without adding extended polymatroid cuts to the formulation as a function of demand correlation. We report the total expected cost, the CPU time in seconds, the percentage integrality gap

Figure 2. Cost and number of DCs as a function of retailers' demand correlation.

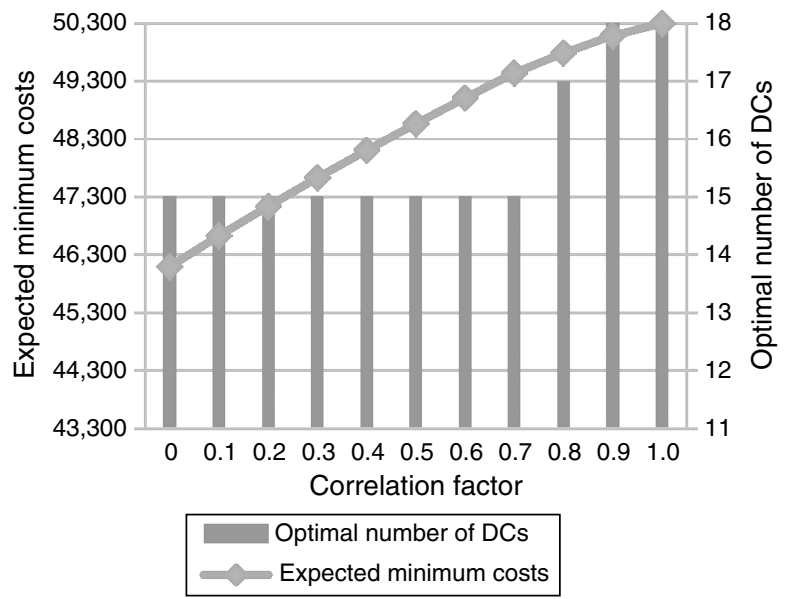

at the root node of the search tree (rgap), and the number of branch-and-bound nodes explored. We observe that computational difficulty increases with higher correlation. However, the cuts are beneficial in reducing the computational burden.

\subsection{Numerical Experiments on Stochastic Lead Time Case}

One of the main effects of considering stochastic lead times in our model is the increment of safety stock cost and, consequently, the increment of expected total cost on the supply chain structure. Table 6 shows this impact under the optimal supply chain design per each case. In particular, we report the number of DCs employed as the lead time standard deviation increases by 0.1 in the 25 -node data set assuming uncorrelated demands. We also report which DCs are opened and closed with respect to the previous run. The number of active DCs increases because the system is capacitated.

Figures 3 and 4 capture the simultaneous impact of retailers' correlated demands and lead time variability on costs and number of DCs, respectively. Hence, we present two three-dimensional graphs that are created from adding a third axis that accounts for lead time variability to the two-dimensional Figure 2. Note that Figure 2 shows the particular case in which the standard deviation of the lead time is 0 . In particular, the retailers' correlation factor $\left(\rho_{i_{1} i_{2}}\right)$ and the lead time standard deviation $\left(\sigma_{L_{j}}\right)$ increase 0.1 per each experiment.

In Figure 3 we observe that the optimal total cost of the supply chain increases when retailers' demand correlation and stochastic lead time variability increased. We go from a value of 46,095 for the uncorrelated and fixed lead time case to a value of 76,129 for the perfectly correlated with 0.5 standard deviation lead time case. This represents a $65 \%$ increase in costs with respect to the uncorrelatedfixed lead time cases versus a raise of the $67 \%$ that would represent to keep the base case supply chain configuration. Figure 4 describes a boost of the number of opened DCs, from 15 DCs to 18, when increments are applied in both directions. Also observe that given the same lead time standard deviation, for the most correlated cases the number of opened DCs is higher compared with lowest correlated cases. This general preference to build new DCs as opposed to keep pooled inventory (i.e., diversification) directly depends on the specific relative parameter values.

\subsection{Numerical Experiments on the Multicommodity Case}

It is interesting to study the scalability of our most general model. To do so, we increase the number of commodities (L) and observe the CPU times and the number of nodes explored in the search tree in two different experiments. Experiment 1 assumes uncorrelated demand between different retailers and different products and a fixed known 
Table 5. Impact of correlation coefficient on solving (CQMIP3).

\begin{tabular}{|c|c|c|c|c|c|c|c|c|}
\hline \multirow[b]{2}{*}{$\rho_{i i^{\prime}}$} & \multirow[b]{2}{*}{ Average cost } & \multicolumn{3}{|c|}{ CPLEX } & \multicolumn{4}{|c|}{ CPLEX + Cuts } \\
\hline & & Time & \% Rgap & Nodes & Time & $\%$ Rgap & Nodes & Cuts \\
\hline 0 & 211,521 & 14 & 0.10 & 7 & 14 & 0.10 & 3 & 2 \\
\hline 0.1 & 213,356 & 32 & 0.32 & 17 & 24 & 0.30 & 4 & 3 \\
\hline 0.3 & 215,429 & 73 & 0.76 & 153 & 89 & 0.75 & 109 & 6 \\
\hline 0.5 & 223,102 & 302 & 2.51 & 710 & 262 & 1.98 & 375 & 11 \\
\hline 0.6 & 228,836 & 844 & 2.47 & 3,204 & 404 & 1.37 & 1,109 & 19 \\
\hline 0.7 & 229,826 & 1,156 & 4.01 & 5,219 & 674 & 2.50 & 1,354 & 21 \\
\hline
\end{tabular}

Table 6. Impact of lead time variability.

\begin{tabular}{|c|c|c|c|c|}
\hline$\sigma_{L_{j}}$ & Cost & DCs & Opened DCs & Closed DCs \\
\hline 0 & 101,868 & 4 & $\begin{array}{l}\text { New York, Los Angeles, } \\
\text { Chicago, Houston }\end{array}$ & - \\
\hline 0.1 & 125,231 & 4 & Philadelphia, Indiana & $\begin{array}{r}\text { New York, } \\
\text { Chicago }\end{array}$ \\
\hline 0.2 & 134,529 & 5 & $\begin{array}{l}\text { New York, San Diego, } \\
\text { Baltimore }\end{array}$ & $\begin{array}{l}\text { Philadelphia, } \\
\text { Los Angeles }\end{array}$ \\
\hline 0.3 & 141,152 & 5 & - & - \\
\hline 0.4 & 147,305 & 5 & $\begin{array}{l}\text { Philadelphia, } \\
\text { San Antonio }\end{array}$ & $\begin{array}{r}\text { New York, } \\
\text { Houston }\end{array}$ \\
\hline 0.5 & 152,090 & 6 & Houston, San Francisco & San Antonio \\
\hline
\end{tabular}

lead time of one at each DC (i.e. block matrices $U$ and $\mathrm{W}$ are diagonal). Experiment 2 assumes nonzeros in all the elements of our block matrices. In particular, there is a correlation of 0.1 between different retailers, 0.1 between different products, and a 0.1 standard deviation of all DCs' lead times. In each run we take the average of 10 random instances based on some randomly generated parameters (refer to Table D.1).

As expected, we observe a better computational performance for experiment 1 compared to experiment 2 , due to sparsity of the correlation matrices and fixed lead time. Overall, we observe a good scalability as a function of $|L|$.

For the 88-node data set when $|L|=3$ (or more), the models become too large (18 million columns, 6 million

Figure 3. Cost as a function of retailers' demand correlation and lead time variability.

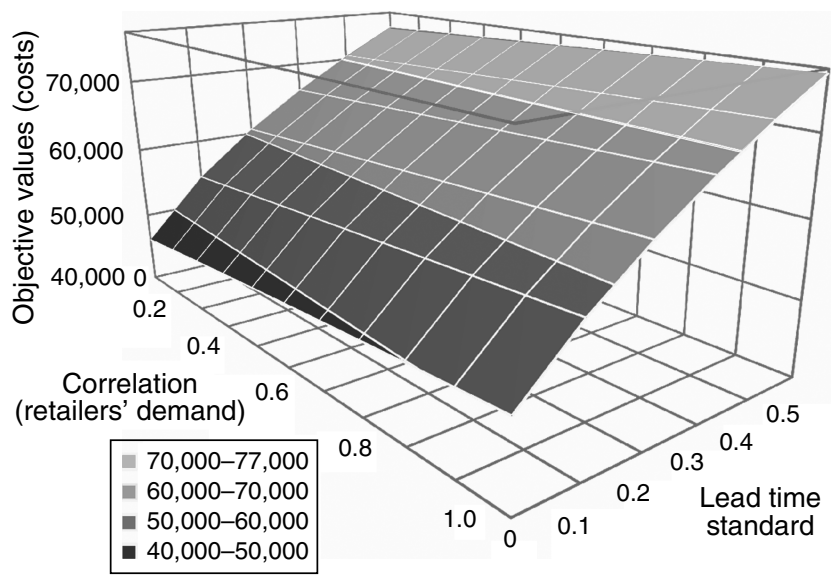

rows, and more than 6 million binary variables) to solve. Our computer runs out of $8 \mathrm{~GB}$ memory during model generation with IBM ILOG Concert Technology. This suggests that for solutions of very large-scale models, column and/or row generation methods would be needed.

Finally, we show an example of the impact of product correlation on the 25-node supply chain with two products. To isolate the correlation effect, we assume that lead time is fixed, retailer demand is uncorrelated, and all DCs are uncapacitated. The latter assumption causes both products to share the same DCs and assignments between DC and retailer. This way we are able to exclusively focus on the

Figure 4. Number of DCs as a function of retailers' demand correlation and lead time variability.

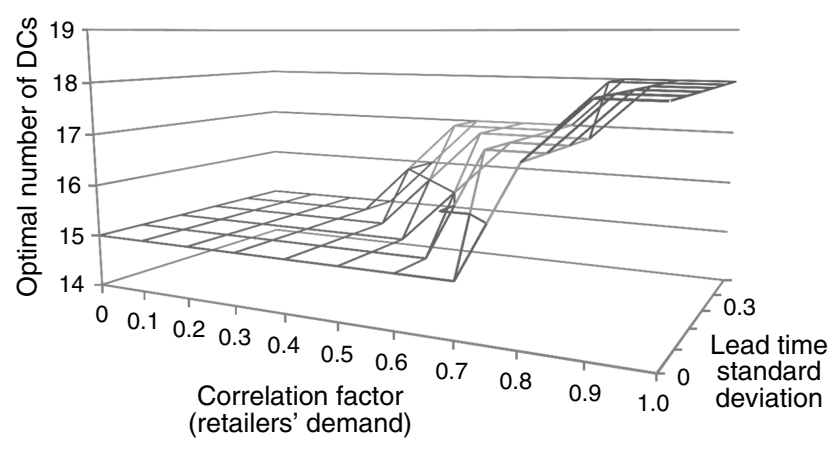

Figure 5. The effect of correlated product demand on the supply chain.

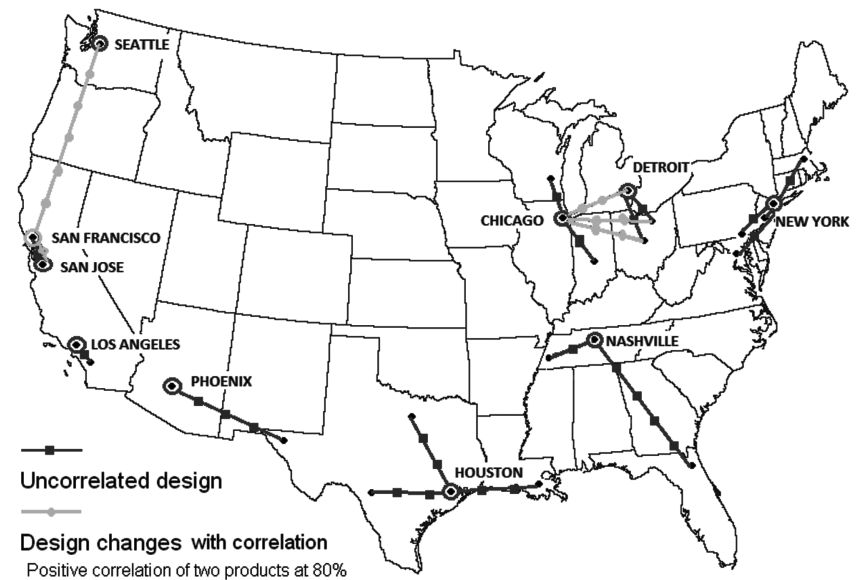


Table 7. Scalability of model 6 with 25 -node data set.

\begin{tabular}{rrccccrrr}
\hline & & & \multicolumn{2}{c}{ Experiment 1} & & \multicolumn{2}{c}{ Experiment 2} \\
$|L|$ & Variables (binary) & Conic constraints & Time & Nodes & & Time & Nodes \\
\hline 1 & $725(650)$ & 50 & 75 & 3 & 0 & 1 & 0 \\
2 & $1,400(1,275)$ & 100 & 14 & 0 & 361 & 0 \\
3 & $2,075(1,900)$ & 125 & 18 & 0 & 256 & 6 \\
4 & $2,750(2,500)$ & 150 & 31 & 0 & 102 & 10 \\
5 & $3,425(3,150)$ & 275 & 173 & 0 & 233 & 5 \\
10 & $6,800(6,275)$ & 400 & 474 & 0 & 695 & 6 \\
15 & $10,175(9,400)$ & & & & & \\
\hline
\end{tabular}

impact of product correlation. The links and circled cities in dark color on Figure 5 show the optimal supply chain design when demand of both products is uncorrelated. In this case, there are nine opened DCs, and the expected total cost is 148,521 . Next, we assume that the products have $80 \%$ correlation. As in the retailer demand correlation case, if we keep the same assignments as in the uncorrelated case, the safety stock levels increase, causing this design to be no longer optimal. If we keep the same design as in the uncorrelated case, total costs would reach 159,062, which represents a $0.35 \%$ increase compared with the new optimal expected total cost of 158,503. DCs in Detroit, Seattle, and San Jose are no longer considered for the new optimal design (in light color). Retailers assigned to Detroit are reassigned to Chicago and a new DC in San Francisco supplies San Jose and Seattle.

Note that in this experiment, positive correlation between different commodities may cause the pooling effect (DCs go from nine to seven) as opposed to the diversified effect that resulted in Figures 2 and 4 when retailers' demand correlation increased. From these two results we can conclude that the decision to build more or fewer DCs compared to the uncorrelated base case depends on the specific tradeoff between fixed location and safety stock costs (DCs are uncapacitated). If location fixed costs are more relevant, a pooling strategy will be considered, and a diversified strategy when the contrary occurs.

\section{Conclusions}

In this paper we describe a conic integer programming approach to stochastic facility location and inventory management models with risk pooling. This new approach not only reasonably leads to similar or better computational solution times than previous column generation and Lagrangian based methods, but more importantly allows one to model more general problems than considered up to now. The solution algorithms developed in Shen et al. (2003), Daskin et al. (2002), and Ozsen et al. (2008) assume that the mean and variance of demand are uniform across retailers. Shu et al. (2005) and Shen and Qi (2007) offer more flexible methods and allow the proportion of retailer mean and variance to be nonuniform. However, these latter references solve the uncapacitated facility problem with uncorrelated demands. In this paper, we remove these restrictive assumptions and consider other novel generalized aspects and still are able to solve the problems efficiently.

We solve each of the models by recasting them as equivalent conic quadratic mixed-integer programs, which can be solved to optimality using commercial software packages. This reduces the burden of developing special purpose algorithms for each special case. However, in cases where optimality cannot be easily reached, we may employ valid cuts to improve the computational performance.

We also make some general recommendations about how best to respond to changes in the model structure. If all demands are uncorrelated, we show that a risk pooling strategy deals effectively with increasing inventory costs. Another situation has been observed when dealing with uncapacitated DCs and incremental positive correlated demands. A diversified location strategy is appropriate when safety stock costs become more relevant than other costs such as fixed location costs. Yet, a pooling strategy can be optimal if fixed costs are relatively higher.

The conic integer programming approach introduced in this paper is versatile and can be applied to other nonlinear supply chain models as well. In particular, our approach could be extended to study pure inventory management models or integrated production and transportation planning models, to name a few. Finally, other integrated location-inventory models with vehicle routing, service, or unreliable supply would benefit from the conic integer programming approach as well.

\section{Appendix}

\section{A. Derivation of Expected Working Inventory}

Following Shen et al. (2003), we describe how the expected working inventory cost at DC $j$ in (P1) is derived. For simplicity, we drop the subscript $j$ from the formulation. The working inventory cost includes the total fixed cost of placing $n$ orders per year $F n$, the shipment cost per year $v(D / n) n$, and the average working inventory cost $(h D) /(2 n)$.

There are $n$ orders per year, and the annual expected demand is $D=\sum_{i \in I} \mu_{i} y_{i j}$. Consider the expression $F n+\beta v(D / n) n+$ $\theta((h D) /(2 n))$. We take the derivative of this expression with respect to $n$ and assume that $v(\cdot)$ is linear $(v(x)=a x+g)$. We obtain $F+\beta g+\beta a(D / n)-\beta a(D / n)-\theta\left((h D) /\left(2 n^{2}\right)\right)=F+$ $\beta g-\theta\left((h D) /\left(2 n^{2}\right)\right)=0$. Solve for $n, n=\sqrt{\theta h D / 2(F+\beta g)}$, and substitute $n$ into the above equation to get $\sqrt{2 \theta h D(F+\beta g)}+$ 
$\beta a D=\sqrt{2 \theta h(F+\beta g)} \sqrt{\sum_{i \in I} \mu_{i} y_{i j}}+\beta a \sum_{i \in I} \mu_{i} y_{i j}$. This expression is part of the objective function in $(\mathrm{P} 1)$.

\section{B. Algorithm to Find Polymatroid Cuts}

The following is the implementation of Edmond's greedy algorithm (Edmonds 1970) for our separation problem. For each $j \in$ $J$, do:

1. Given $y_{j}^{*} \in[0,1]^{|I|}$ and $t_{j}^{*}$, sort $y_{i j}^{*}$ in nonincreasing order

$y_{(1) j}^{*} \geqslant y_{(2) j}^{*} \geqslant \cdots$.

2. For $i=1, \ldots,|I|$, let $S_{i}=\{(1),(2), \ldots,(i)\}$ and $\pi_{(i)}=$ $\sqrt{\sum_{k \in S_{i}} \sigma_{(k)}^{2}}-\sqrt{\sum_{k \in S_{i-1}} \sigma_{(k)}^{2}}$.

3. If $\zeta_{j}=\pi y_{j}^{*}>t_{j}^{*}$, we add the extended polymatroid cut $\pi y_{j} \leqslant t_{j}$ to the formulation.

\section{Heuristic to Find Cover Cuts}

The following is the implementation of Atamtürk and Narayanan's cover inequality separation algorithm (Atamtürk and Narayanan 2009) for our problem. Let $\bar{y}_{i j}=1-y_{i j}^{*}$ for $i \in I, j \in J$. For each $j \in J$ and for each distinct pair $i_{1}$ and $i_{2}$ in $I$ do:

1. Solve the following system of equations on variables $\lambda$ and $\rho$ :

$\bar{y}_{i_{1} j}=L_{j} \mu_{i_{1}} \lambda+z_{\alpha}^{2} L_{j} \sigma_{i_{1}}^{2} \rho$,

$\bar{y}_{i_{2} j}=L_{j} \mu_{i_{2}} \lambda+z_{\alpha}^{2} L_{j} \sigma_{i_{2}}^{2} \rho$.

2. If $(\lambda, \rho) \geqslant 0$, then sort each $i$ in nondecreasing order of $\bar{y}_{i j} /\left(L_{j} \mu_{i_{1}} \lambda+z_{\alpha}^{2} L_{j} \sigma_{i}^{2} \rho\right)$; that is,

$\frac{\bar{y}_{(1) j}}{L_{j} \mu_{(1)} \lambda+z_{\alpha}^{2} L_{j} \sigma_{(1)}^{2} \rho} \leqslant \frac{\bar{y}_{(2) j}}{L_{j} \mu_{(2)} \lambda+z_{\alpha}^{2} L_{j} \sigma_{(2)}^{2} \rho} \leqslant \cdots$.

3. Assign $z_{(i)}=1$ following the established order until $z_{\alpha} \sqrt{L_{j}} \sqrt{\sum_{i \in I} \sigma_{i}^{2} z_{i j}}+L_{j} \sum_{i \in I} \mu_{i} z_{i j}>C_{j}$.

4. If $\zeta=\bar{y} z<1$, then we add the cover cut $\sum_{i \in S} x_{i} \leqslant|S|-1$ to the formulation, where $S$ is the ground set for $z$.

\section{Parameter Values}

Table D.1. Parameters used in all experiments.

Parameter Value

Used in all experiments

$d_{i j}$

$F_{j}, g_{j}$

$a_{j}$

$h, \chi, L$

$\alpha$

$z_{\alpha}$

Great circle distance

10

5

0.975

1.96

Table 1 ( 88,150 nodes) $f_{j}$

$\mu_{i}, \sigma_{i}^{2}$
From Daskin (1995) divided by 100 , if 88 nodes 100 , if 150 nodes

Demand 1 from Daskin (1995) divided by $1,000, \mu_{i}$
Table D.1. Continued

Table 3 (15, 88, 150 nodes)

$f_{j}$

$\mu_{i}, \sigma_{i}^{2}$

$(\beta, \theta)$

From Daskin (1995) divided by 100 , if 15 or 88 nodes 100,000 , if 150 nodes

Description in Özsen et al.

(2008), $\mu_{i}$

$(0.00001,0.001)$

Table 4 (88 nodes)

$f_{j}$

$\mu_{i}, \sigma_{i}^{2}$

$(\beta, \theta)$

Uniform [40,000, 50,000]

Description in Özsen et al. (2008) $\left(1+\epsilon_{i}\right), \mu_{j}\left(1+\epsilon_{i}\right)$

$(0.0004,10)$

Figure 1 (25 nodes)

$f_{j}$

$\mu_{i}, \sigma_{i}$

$(\beta, \theta), C_{j}$

10,000

Demand 1 from Daskin (1995),

demand 2 from Daskin (1995)

(0.00001, 0.001), 17,000,000

Figures 2, 3, 4 (25 nodes)

$f_{j}$

$\mu_{i}, \sigma_{i}$

$(\beta, \theta), C_{j}$

Table 5 (25 nodes)

$f_{j}$

$\mu_{i}, \sigma_{i}$

$(\beta, \theta), C_{j}$

1,000

Demand 1 from Daskin (1995), demand 2 from Daskin (1995)

(0.00001, 0.001), 200,000,000

Uniform [40,000, 50,000]

Demand 1 from Daskin (1995) $\left(1+\epsilon_{i}\right)$, demand 2 from Daskin $(1995)\left(1+\epsilon_{i}\right)$

(0.00001, 0.0001), 17,500,000

Table 6 ( 25 nodes)

$$
\begin{array}{ll}
f_{j} & 10,000 \\
\mu_{i}, \sigma_{i} & \begin{array}{c}
\text { Demand 1 from Daskin (1995), } \\
\text { demand 2 from Daskin (1995) } \\
(\beta, \theta), C_{j}
\end{array} \\
(0.00001,0.001), 17,000,000
\end{array}
$$

Table 7 ( 25 nodes)

$$
\begin{aligned}
& f_{j} \\
& \mu_{i l}, \sigma_{i l}
\end{aligned}
$$

Uniform [40,000, 50,000]

Demand 1 from Daskin (1995) $\left(1+\epsilon_{i l}\right)$, demand 2 from Daskin $(1995)\left(1+\epsilon_{i l}\right)$

$(\beta, \theta), C_{j}$

$(0.001,0.001), 2,000,000,000$

Figure 5 (25 nodes)

$$
\begin{aligned}
& f_{j} \\
& \mu_{i 1}, \sigma_{i 1}^{2} \\
& \mu_{i 2}, \sigma_{i 2}^{2}
\end{aligned}
$$$$
(\beta, \theta), C_{j}
$$

6,000

Demand 1 from Daskin (1995) divided by $100, \mu_{i 1}$

Demand 2 from Daskin (1995)

divided by $100, \mu_{i 2}$

$(0.001,0.1), 2,000,000,000$

\section{Acknowledgments}

The authors thank Lian Qi and Leyla Özsen for sharing their computer programs with the authors. They are also grateful to the anonymous referees and the associate editor for their insightful comments. This research was partially supported by the National Science Foundation [Grant CMMI 1068862].

\section{References}

Alizadeh, F., D. Goldfarb. 2003. Second-order cone programming. Math. Programming 95(1) 3-51. 
Atamtürk, A., V. Narayanan. 2008. Polymatroids and mean-risk minimization in discrete optimization. Oper. Res. Lett. 36(5) 618-622.

Atamtürk, A., V. Narayanan. 2009. The submodular knapsack polytope. Discrete Optim. 6(4) 333-344.

Atamtürk, A., V. Narayanan. 2010. Conic mixed-integer rounding cuts. Math. Programming 122(1) 1-20.

Atamtürk, A., V. Narayanan. 2011. Lifting for conic mixed-integer programming. Math. Programming 126(2) 351-363.

Ben-Tal, A., A. S. Nemirovski. 2001. Lectures on Modern Convex Optimization: Analysis, Algorithms, and Engineering Applications, Vol. 2. Society for Industrial and Applied Mathematics, Philadelphia, PA.

Çezik, M. T., G. Iyengar. 2005. Cuts for mixed 0-1 conic programming. Math. Programming 104(1) 179-202.

Charnes, J. M., H. Marmorstein, W. Zinn. 1995. Safety stock determination with serially correlated demand in a periodic-review inventory system. J. Oper. Res. Soc. 46(8) 1006-1013.

Dasci, A., V. Verter. 2001. The plant location and technology acquisition problem. IIE Trans. 33(11) 963-974.

Daskin, M. S. 1995. Network and Discrete Location: Models, Algorithms and Applications. Wiley, New York.

Daskin, M. S., C. R. Coullard, Z. J. M. Shen. 2002. An inventory-location model: Formulation, solution algorithm and computational results. Ann. Oper. Res. 110(1) 83-106.

Edmonds, J. 1970. Submodular functions, matroids, and certain polyhedra Combin. Structures and Their Appl. 11 69-87.

Erkip, N., W. H. Hausman, S. Nahmias. 1990. Optimal centralized ordering policies in multi-echelon inventory systems with correlated demands. Management Sci. 36(3) 381-392.

Fine, C. H., R. M. Freund. 1990. Optimal investment in product-flexible manufacturing capacity. Management Sci. 36(4) 449-466.

Geoffrion, A. M., G. W. Graves. 1974. Multicommodity distribution system design by Benders decomposition. Management Sci. 20(5) 822-844.
Goyal, M., S. Netessine. 2011. Volume flexibility, product flexibility or both: The role of demand correlation and product substitution. Manufacturing Service Oper. Management 13(2) 180-193.

Inderfurth, K. 1991. Safety stock optimization in multi-stage inventory systems. Internat. J. Production Econom. 24(1-2) 103-113.

Johnson, G. D., H. E. Thompson. 1975. Optimality of myopic inventory policies for certain dependent demand processes. Management Sci. 21(11) 1303-1307.

Kuo, Y. J., H. D. Mittelmann. 2004. Interior point methods for secondorder cone programming and OR applications. Comput. Optim. Appl. 28(3) 255-285.

Nahmias, S. 1993. Production and Operations Analysis. Irwin Professional Publishing, Homewood, IL.

Özsen, L., C. R. Coullard, M. S. Daskin. 2008. Capacitated warehouse location model with risk pooling. Naval Res. Logist. 55(4) 295-312.

Shen, Z. J. M. 2005. A multi-commodity supply chain design problem. IIE Trans. 37(8) 753-762.

Shen, Z. J. M. 2007. Integrated stochastic supply chain design models. Comput. Sci. Engrg. 9(2) 50-59.

Shen, Z. J. M., M. S. Daskin. 2005. Trade-offs between customer service and cost in integrated supply chain design. Manufacturing Service Oper. Management 7(3) 188-207.

Shen, Z. J. M., L. Qi. 2007. Incorporating inventory and routing costs in strategic location models. Eur. J. Oper. Res.179(2) 372-389.

Shen, Z. J. M., C. Coullard, M. S. Daskin. 2003. A joint location-inventory model. Transportation Sci. 37(1) 40-55.

Shu, J., C. P. Teo, Z. J. M. Shen. 2005. Stochastic transportation-inventory network design problem. Oper. Res. 53(1) 48-60.

Snyder, L. V., M. S. Daskin, C. P. Teo. 2007. The stochastic location model with risk pooling. Eur. J. Oper. Res. 179(3) 1221-1238.

Qi, L., Z. J. M. Shen. 2007. A supply chain design model with unreliable supply. Naval Res. Logist. 54(8) 829-844. 